\title{
Video Article \\ Updated Technique for Reliable, Easy, and Tolerated Transcranial Electrical Stimulation Including Transcranial Direct Current Stimulation
}

\author{
Helen Borges ${ }^{1}$, Alexandra Dufau ${ }^{1,2}$, Bhaskar Paneri ${ }^{1}$, Adam J. Woods ${ }^{3}$, Helena Knotkova ${ }^{4,5}$, Marom Bikson ${ }^{1}$ \\ ${ }^{1}$ Department of Biomedical Engineering, The City College of New York, CUNY \\ ${ }^{2}$ Department of Clinical and Health Psychology, Center for Cognitive Aging and Memory \\ ${ }^{3}$ McKnight Brain Institute, University of Florida \\ ${ }^{4}$ MJHS Institute for Innovation in Palliative Care \\ ${ }^{5}$ Department of Family and Social Medicine, Albert Einstein College of Medicine
}

Correspondence to: Marom Bikson at bikson@ccny.cuny.edu

URL: https://www.jove.com/video/59204

DOI: doi:10.3791/59204

Keywords: Medicine, Issue 155, Transcranial Direct Current Stimulation, tDCS, Neuromodulation, Direct Current Modulation, Safety, Noninvasive Brain Stimulation

Date Published: $1 / 3 / 2020$

Citation: Borges, H., Dufau, A., Paneri, B., Woods, A.J., Knotkova, H., Bikson, M. Updated Technique for Reliable, Easy, and Tolerated Transcranial Electrical Stimulation Including Transcranial Direct Current Stimulation. J. Vis. Exp. (155), e59204, doi:10.3791/59204 (2020).

\section{Abstract}

Transcranial direct current stimulation (tDCS) is a noninvasive method of neuromodulation using low-intensity direct electrical currents. This method of brain stimulation presents several potential advantages compared to other techniques, as it is noninvasive, cost-effective, broadly deployable, and well-tolerated provided proper equipment and protocols are administered. Even though tDCS is apparently simple to perform, correct administration of the tDCS session, especially the electrode positioning and preparation, is vital for ensuring reproducibility and tolerability. The electrode positioning and preparation steps are traditionally also the most time consuming and error-prone. To address these challenges, modern tDCS techniques, using fixed-position headgear and pre-assembled sponge electrodes, reduce complexity and setup time while also ensuring that the electrodes are consistently placed as intended. These modern tDCS methods present advantages for research, clinic, and remote-supervised (at home) settings. This article provides a comprehensive step-by-step guide for administering a tDCS session using fixed-position headgear and pre-assembled sponge electrodes. This guide demonstrates tDCS using commonly applied montages intended for motor cortex and dorsolateral prefrontal cortex (DLPFC) stimulation. As described, selection of the head size and montage-specific headgear automates electrode positioning. Fully assembled pre-saturated snap-electrodes are simply affixed to the set position snap-connectors on the headgear. The modern tDCS method is shown to reduce setup time and reduce errors for both novice and expert operators. The methods outlined in this article can be adapted to different applications of tDCS as well as other forms of transcranial electrical stimulation (tES) such as transcranial alternating current stimulation (tACS) and transcranial random noise stimulation (tRNS). However, since tES is application specific, as appropriate, any methods recipe is customized to accommodate subject, indication, environment, and outcome specific features.

\section{Video Link}

The video component of this article can be found at https://www.jove.com/video/59204/

\section{Introduction}

Transcranial direct current stimulation (tDCS) is a noninvasive brain stimulation technique capable of modulating cortical excitability ${ }^{1,2}$. During tDCS, a constant low intensity current, typically 1-2 milliamperes (mA), flows from an anode electrode to a cathode electrode generating a weak electric field across the cortex ${ }^{3,4}$. Conventional tDCS protocols are considered tolerated and safe ${ }^{5}$. The effects of one session of tDCS can last several minutes after session completion ${ }^{6}$ with repeated sessions producing longer lasting changes in brain function ${ }^{7,8}$. The tolerability profile and the potential to produce either acute or long-lasting changes makes tDCS a candidate for a variety of interventions and treatments ${ }^{9,10,11}$. While questions remain about the optimal dose of $\operatorname{tDCS}^{12}$, including the role of intensity ${ }^{13}$, polarity ${ }^{7}$ and focality ${ }^{3}$, the importance of controlling electrode placement for neuromodulation reproducibility is accepted. Moreover, electrode preparation also underpins tolerability and related concerns such as blinding-reliability ${ }^{14}$. While tDCS has practical advantages over other brain stimulation methods, due to its cost-effectiveness, portability, ease of use, and tolerability; nonetheless, the apparent simplicity and adaptability of the technique does not excuse poor electrode preparation and placement technique ${ }^{14}$

Indeed, the apparent simplicity of tDCS has, in some cases, encouraged insufficient attention to proper equipment, supplies, and operator training $^{14}$. First, reliable electrode placement is required for reproducibility. The positioning of tDCS electrodes on the scalp typically follows the 10-20 system, which is a method used for the placement and application of electroencephalography (EEG) electrodes. In the conventional tDCS method, this involves tape measurement to establish electrode location, with several measurements at every session ${ }^{15,16,17}$. A marker is used to label scalp positions. There is potential for this process to result in electrode placement variability (e.g., how reliably various operators position measuring tape), especially under high throughput conditions - though rigorous operator training and certification can mitigate variability. In the conventional tDCS method, the electrodes are then manually pressed onto the measured coordinate and rubber-straps applied in an ad 
hoc manner ${ }^{18}$ (e.g., the tightness of bands may not be consistent across operators affecting ejection of fluid from sponges, subject tolerability, and even drift in electrode position ${ }^{19,20}$ ). As with electrode position, this variability can be mitigated with explicit protocols and training, though such detail is often not described in published reports. In special circumstances when the pad electrode is separated from the scalp by cream/ gel without the use of sponge ${ }^{21}$, caution is required to prevent direct electrode-skin contact leading invariably to a burn ${ }^{14}$. An alternative less common method for tDCS uses an elastic cap ${ }^{22,23}$, which depends on subject specific head deformation not distorting electrode position, and risks saline spread and bridging under the cap (not visible to the operator). In comparison to conventional rubber-band or elastic-cap based techniques, the modern tDCS technique presented here makes the critical electrode preparation and positioning steps more robust and reliable.

Another key procedure in tDCS is the assembly of the electrodes. Conventional tDCS electrodes are multi-part. These separate parts, which have to be assembled carefully by the operator, consist of metal or conductive-rubber electrodes, which the operator encloses in a perforated sponge pocket and saturates with saline solution ${ }^{15}$. While not complex, the process of electrode assembly requires training and vigilance at each session, as a small error such as metal/rubber protruding from the sponge and contacting the subject or saline fluid volume can lead to skin injury ${ }^{14}$. The modern tDCS technique overcomes these concerns by use of pre-assembled pre-saturated electrodes/sponges that moreover include a reliable snap connector to the headgear. Pre-assembled and pre-saturated electrodes are single use, mitigating issues of reproducibility and risks of contamination with reused sponges ${ }^{14,20}$.

The purpose of this article is to demonstrate modern setup procedures for the administration of tDCS and related transcranial electrical stimulation techniques, such as transcranial alternating current stimulation (tACS), transcranial ransom noise stimulation (tRNS) ${ }^{24}$, and transcranial pulsed current stimulation (tPCS) and its variants ${ }^{25}$. This guide demonstrates tDCS using commonly applied montages intended for motor cortex ${ }^{26}$ and dorsolateral prefrontal cortex (DLPFC) stimulation ${ }^{27}$. The modern tDCS technique explained here avoids tape measurement for determining electrode placement, cumbersome carbon-rubber electrode insertion, tedious procedure of wetting electrode sponges, and use of rubber bands or elastic caps as headgear. This process is optimized by using a specialized fixed-position headgear and a pre-saturated snap connector electrode. The fixed-position headgear consists of straps deigned to automatically place tDCS electrodes at standard 10-10 EEG ${ }^{19}$. The pre-determined electrode location provided by these straps removes the need for extensive measuring and calculations, thus increasing reproducibility, time-effectiveness and subject manipulation. Only a one-time fitting measurement is needed (used to determine the correct strap size to be used) at the first visit. Single use pre-assembled sponge electrodes are provided pre-soaked in the optimized volume of saline and with the rubber electrode inserted and fixed, minimizing the risk of direct contact between the rubber/metal and the skin, as well as over/ under-soaking. Using fixed-position headgear and pre-assembled sponge electrodes (Figure 1) not only significantly reduces the possibility for electrode misplacement due to measurement error, but also make administering tDCS easier and more time-effective. For each montage, there is a specific headgear. This article will use two montages as examples. The first montage is the M1-SO in which the anode is placed over the region corresponding to primary motor cortex (M1) and the cathode is placed over the contralateral supra-orbital (SO) region (Figure 2A). The second montage is the bifrontal montage, in which the anode is placed over the right and the cathode is placed over the left DLPFC (F3/ F4, Figure 2C). The methods outlined here are not limited to the aforementioned montages, and can be adapted to the other configurations, significantly reducing the possibility for electrode misplacement due to measurement error, while also making the application of tDCS and related tES techniques more efficient. Modern headgears described here are electrode montage specific (e.g., M1-SO, F3/F4) and different headgear would be used for separate electrode montages. Even though, the modern technique reduces the number of steps and makes the administration of $\mathrm{tES}$ technique efficient, the new approach still requires training to operate the stimulator.

\section{Protocol}

The City College of New York, CUNY Institutional Review Board (IRB) approved this protocol.

\section{Materials}

1. Prior to the tDCS session, make sure that all necessary materials are available. While some materials will depend on the specific protocol of the study/treatment, there are basic items that are general across modern tDCS application as shown here (Table 1, Figure 3).

1. Prepare a tDCS device: A battery-driven tDCS device that functions as a constant current stimulator with a maximum output in the milliamp range. A tES device with a tDCS setting may be used (e.g., Soterix Medical 1x1 tES device).

2. Prepare single-use snap sponge electrodes (e.g., Soterix Medical $5 \times 5 \mathrm{~cm}$ snap electrodes).

3. Prepare saline solution and applicator, to be used if the electrode becomes dehydrated during the session. Since pre-assembled electrodes are already soaked with a volume of saline solution pre-determined to be sufficient, a minimal amount of saline, if any, might be added. Be careful not to oversoak the sponge and avoid leakage and dripping by gradually and carefully adding saline only if needed.

2. Prepare fixed-position headgear. Here, two models of snap-headgear are used (M1-SO and bifrontal).

3. Prepare connecting cables. The snap-headgear already includes the necessary cables, which one end configured to connect to the stimulation (male banana) and the other end configured to accept the snap pad (female snap). This may differ according to the fixed-position headgear chosen.

4. Prepare relevant forms (e.g. consent form, pre- and post-questionnaires, screening forms, data collection forms) and other interventionspecific materials as applicable.

\section{Relevant forms}

1. When the subject arrives, first greet the subject, and then have him or her sit comfortably in an upright position in a chair.

2. For research trials, before the study, have the subject give consent to participate in the study. The consent form includes details about the research protocol, risks, and benefits of the study. This form is intended to disclose appropriate information to subjects so that they may make a voluntary choice to accept or refuse treatment. It originates from legal and ethical rights. A subject must be aware of what happens to his or her body, and the ethical responsibilities of a researcher to get the participant involved in his or her physical and mental well-being. 
3. For research trials, collect a written consent from the participants before any study procedures are performed. Show the consent form to the subject. An experiment can only continue if the subject chooses to sign the consent form.

4. Screen the subject according to the inclusion and exclusion criteria outlined in the study's protocol.

5. If no contraindications are present and the subject still agrees to participate, ask subject to fill out any other necessary forms (i.e., demographics form, relevant pre-questionnaires, etc.)

6. If subject fully understands and consents to the procedure to follow and has filled out the necessary forms, go on to the next step.

\section{Measurements}

1. Begin setup by first measuring the subject's head circumference to determine the appropriate size of headgear to be used. To measure the subject's head circumference, start from the most prominent part of the forehead around the widest part of the back of the head, going over the hair and above the ears. Fixed-position head bands require significantly less measurements than the conventional methods of electrode placement for $\mathrm{tDCS}^{15}$ and moreover only require measurement at the first visit when the head-gear is selected.

NOTE: Different headgears may vary in the range of sizes offered as well as in the circumference measurements corresponding to each size. For the headgear used in this demonstration, the sizes available are small $(52-55.5 \mathrm{~cm})$, medium $(55.5-58.5 \mathrm{~cm})$, large $(58.5-62 \mathrm{~cm})$, and extra-large $(62-65 \mathrm{~cm})$.

2. With the subject sitting comfortably in a chair, proceed to measure the head circumference to determine the appropriate size of the headgear.

3. Consult the specific headgear manual to select the appropriate headgear size (e.g., small, medium, large) based on the desired electrode montage and the subject's head circumference. For most electrode montages, there may be different sized of headgear depending on the subject head size.

\section{Skin preparation}

1. Inspect the skin where the electrode is expected to be placed. In this protocol, place electrodes following either the M1-SO or the bifrontal montage. If any lesions are observed, do not administer tDCS.

2. Ensure the area is free of signs of lotion, dirt, etc.

3. In traditional approaches where re-usable electrodes are used, inspect the rubber inserts and sponges for wear at every session. Here, in modern approach with single-use electrodes, this step is not strictly required. None the less, inspect new electrodes for integrity and saturation.

\section{Electrode placement}

1. Remove two pre-saturated $5 \mathrm{~cm} \times 5 \mathrm{~cm}$ snap electrodes from their packets.

2. Snap the single-use snap electrodes onto the snap-headgear according to the fixed locations on the headgear. These locations are montagespecific and based on the headgear selected. The montage used is study-specific.

3. Optionally, gently expose the scalp by parting the subject's hair with fingers to assure that the saline seeps through the hair into the scalp, enhancing contact quality between the electrode and the scalp.

4. Making sure that the sponge is secured to the strap, position the headgear on the subject's head.

1. In the M1SO snap-headgear montage with "anodal" stimulation of M1, position the anode near the motor cortex and the cathode over the supraorbital area. To accurately position the electrodes onto their designated scalp positions, first position the nasion-representing ring of the strap, located at the bottom portion of the strap, over the nasion. The nasion is the point anterior to the brain, located between the forehead and the nose. Adjust the top portion of the strap so that it is perpendicular to the bottom portion of the strap. The top portion of the strap is intended to sit approximately above the ear, symmetrically positioned on both sides of the head. Then, position the posterior elastic portion of the strap over the inion. The anode/cathode polarity may be reversed depending in application.

2. In the bifrontal (F3/F4) snap-headgear montage with "anodal" stimulation of the left DLPFC, position the anode near the left dorsal lateral prefrontal cortex and the cathode near the right dorsal lateral pre-frontal cortex. The anode/cathode polarity may be reversed depending in application.

5. In some subjects with long hair, ask the subject to tie their hair back or secure the hair tightly while the headgear is being placed. This will allow for a more consistent electrode setup and decrease the risk of discomfort caused by accidental tugging of the subject's hair.

NOTE: Long hair may also present a barrier for fluid from the electrode to saturate to the scalp, and can be gently parted under the electrode.

6. Ensure that the headgear is snug, but not uncomfortably tight. Select the correct size of headgear that does not cause discomfort to the subject while ensuring the sponge electrodes are held reliably to the scalp.

7. Connect the black cable (cathode) and red cable (anode) to the tES device. Consult the operating manual for the stimulator in order to establish whether the stimulator is powered on before or after connecting positioned electrodes to the stimulator.

1. While the stimulator is active, ensure that the electrodes are connected when current flow has been initiated.

8. For the snap-headgear, connect the black cathode cable into the corresponding input black driver of the tDCS device and repeat this for the red anode cable for its respective location on the tDCS device. Ensure the connection polarity is correct as the effects of tDCS are polarity specific.

NOTE: When using a tDCS device, the anode electrode is the positive terminal where positive current enters the body, and the cathode electrode is negative terminal where positive current exits the body. When using a tACS device, anode and cathode are not considered as positive or negative, as both terminals will act anode and cathode alternatively. Conventionally, red indicates the anode electrode, and black or blue indicates the cathode electrode (ensure that the same applies for the device being used).

\section{Start tDCS}

1. Before initiating the tDCS session, make sure that the subject is comfortable and awake. 
2. Confirm that the device is turned on, the cables are properly connected, and the headgear and electrode properly situated. The impedance meter is a secondary method to ensure good contact, but it does not replace the need to make sure all protocol steps are adhered to.

3. Check the impedance meter for contact quality. The device used in this demonstration displays impedance information in real time. This may be device-specific, so become familiar with the impedance meter on the device used.

1. If the subject's overall contact quality is abnormally low, this may indicate improper electrode setup, resulting in high impedance. If the contact quality continues to be low after adjusting the headgear and/or judiciously supplementing saline, press "pre-stim tickle" (if available on the device being used) to achieve a better contact quality.

4. Check if the device has enough battery. Devices designed for tDCS trials have a readily visible low-battery warning - for the device used here directly above the on/off switch there is a low-battery alert indicator.

5. Program the tDCS session duration, intensity or (if applicable to device being used) sham condition setting (for studies with operatory blinding regarding the sham vs real tDCS condition, the setting will be programmed by independent personnel or pre-coded into the device ${ }^{28}$ ). Note that some stimulators are recommended to be switched on before the contact between the electrodes and the skin is made.

1. If the tDCS session is being administered using a tES device, select the tDCS waveform setting.

2. When applying a tES waveform other than tDCS, such as tACS or tPCS, make sure that the device is properly programmed including waveform and frequency.

6. Initiate the tDCS by pressing the Start button. In order to reduce any adverse effects, devices include automatic current ramp up at the initiation of stimulation, along with an automatic ramp down at the end. At the beginning of stimulation, subjects will often perceive an itching and/or tingling sensation underneath the electrodes, which then fades out in most cases.

7. As some subjects may experience discomfort during the first few minutes of tDCS, moderately decrease the current by using the Relax knob temporarily as the subject adjusts. Then, gradually increase the current back up to the desired level. This feature may depend on the device being used and protocol.

1. Ensure that the subject does not touch the device, headgear, and/or electrodes during the stimulation session. Ensure that any required adjustments to these are handled by the operator only.

2. For some subjects, sudden changes in current intensity may produce dizziness or vertigo as well as retinal phosphines if the current is suddenly increased or decreased. To avoid these adverse sensations, make sure to allow a ramp-up and ramp-down time for the stimulation. As previously mentioned, tDCS devices offer an automatic ramp-up/down period. Check the device for specific details.

8. Ensure that the subject remains comfortable and avoids unnecessary movement.

9. If the electrodes become dehydrated, as may be indicated by a decrease in contact quality, use a syringe to gradually add a measured amount of saline to the electrodes. There may be experimental plans where tDCS electrodes are positioned on the head well in advance of stimulation such that when stimulation is scheduled to start the electrodes have been on the head for some time and may become dehydrated.

NOTE: Electrodes designed for tDCS, such as snap electrodes have been developed by the manufacturer to maintain saturation during the course of a tDCS session (e.g., tens of minutes). However, certain environments (such as exceptionally dry atmosphere of air conditioning) may accelerated electrode dehydration. Snap electrodes are pre-saturated, so the need for additional saline is minimized.

1. To avoid saline drip due to gravity, ensure graded application to the top edge of the sponges.

2. To minimize dehydration, avoid an extensive long time between tDCS setup and start of tDCS or if unavoidable (a long task that must be conducted after headgear application but before tDCS application), add checks to confirm sponge saturation and impedance.

10. Avoid touching the electrodes during stimulation. If addition of saline does not improve the contact quality, confirm skin sensation from the subject. Every trial and device will have explicitly specific criteria for any headgear or electrode adjustment steps before or during tDCS including when stimulation is aborted based on impedance and/or subject sensation.

11. At the end of the stimulation session, the device will ramp down from the treatment intensity to $0 \mathrm{~mA}$. Do not allow subject to remove headgear themselves. Do not remove headgear before the device indicates stimulation is complete with a current of zero. As the current ramps down, some subjects may report increased sensations such as tingling. These minor sensations stop after the current intensity returns to zero.

12. When the device has finished ramping down and the current is zero, turn off the device.

\section{After the procedure}

1. Remove headgear loaded with the electrodes from subject's scalp.

2. Disconnect the snap electrodes from the strap. Dispose of the snap electrodes (as they are single use)

3. Inspect the skin under the electrodes. Mild to moderate redness is expected during tDCS ${ }^{5,11,29}$, most of it simply from pressure ${ }^{30}$.

4. Administer a questionnaire of adverse events to assess possible side-effects. Adverse event questionnaires can include any adverse effects typically associated with tDCS, such as tingling, itching and burning sensations, headache and discomfort. Examples for such a questionnaire can be found in Brunoni et al. (2011) ${ }^{31}$.

5. Though tDCS is consider safe when following standard protocols ${ }^{5}$, perform an adverse-event monitoring procedure during the development of any study's protocol. Especially in some patient populations, serious adverse event may occur unrelated to tDCS. Adverse-event monitoring procedures include a course of action to be followed if the subject reports unexpected or serious side-effects during or after the session. Follow the adverse-event monitoring procedures closely and carefully.

Representative Results

The modern tDCS methods described in the guide is expected to simplify tDCS setup and so reduce preparation time while increasing reliability. Setup times were measured using the traditional and modern tDCS methods. Separate consideration was given for experts vs. novices for each method $(n=8)$. Each novice or expert operator conducted the setup five times. For tDCS traditional method both experts and novices reviewed preparation instructions ${ }^{15}$, as well as additional instructions before the first setup trials. For the modern tDCS method, both experts and novices 
reviewed an earlier version this guide. In all cases, operators were allowed to ask observers questions and for instructions as needed, which would be factored into setup time. Observers did not otherwise provide feedback. Reliability was scored by the observer after each trial on a 1-3 scale as: (1) Poor setup with substantial error in electrode placement $(>5 \mathrm{~cm})$ and/or significant uneven electrode contact with skin $(>50 \%$ of sponge surface not contacting skin), and/or other significant errors; (2) Moderate or small error in electrode placement (3-5 cm) and/or moderate uneven electrode contact with skin (30-50\% of sponge surface not contacting skin), and/or other minor errors; (3) No evident error in electrode placement or significant uneven electrode contact with skin, and no other significant errors.

\section{Traditional Method}

The traditional method requires measurements for the M1-SO position before each application using the measurement protocol based the 1020 EEG system. Sponges needed to be assembled and saturated. The novice operators were given an instruction manual with directions for the measurement of the 10-20 EEG system, which they could read before the trial. This instruction manual was kept during the trials for reference. Both expert and novice completed 5 setup trials including the required head-measurements at every trial. The individual times taken for each setup trial were recorded (Figure 4). The average setup time taken by the expert was 7.93 minutes $( \pm 2.30)$. The average setup time taken by the novice was 10.47 minutes $( \pm 3.36)$. Novices were generally unable to achieve an error free setup even at the 5 th session. Experts made infrequent setup errors.

\section{Modern Method}

The modern methods require the head circumference of each subject is measured once in order to determine the appropriate size of the headgear to be used (S: $52-55.5 \mathrm{~cm}, \mathrm{M}: 55.5-58.5 \mathrm{~cm}$, L: $58.5-62 \mathrm{~cm}, \mathrm{XL}: 62-65 \mathrm{~cm}$ ). Sponges were pre-assembled and pre-saturated. The individual times taken for each setup trial were recorded (Figure 4). The average setup time taken by the expert was 1.23 minutes $( \pm 0.37)$. The average setup time taken by the novice was 2.53 minutes $( \pm 0.48)$. Novices were generally achieved an error free setup by the 5 th sessions and any errors were minor. Experts made no setup errors. The modern tDCS approach here increases setup reliability while decreasing stimulation setup time.

\section{Position Error}

The modern tDCS method allows electrode placement with comparable precision to an expert operator measuring traditional EEG 10-10 position. For example, for the M1-S0 using an appropriately designed strap, the mean position error is $1.5 \mathrm{~mm}$, which is significantly less than the electrode size $(5 \mathrm{~cm} \times 5 \mathrm{~cm})$ and not a relevant error for underling brain current flow ${ }^{19}$. For operator or self-application, the modern tDCS method is highly reliable.

\section{Deployability}

The modern tDCS method can be as part of a tele-health program for chronically ill patient with multiple symptoms, including palliative care. For the M1-SO montage, replicable electrode placement was achieved. There were no difficulties with patients' training, protocol adherence, or tolerability ${ }^{26}$. For the bifrontal montage replicable and tolerable stimulation was achieved in both patients with multiple sclerosis and Parkinson's disease $^{32}$, confirming reliable placement was achieved even for self-application in subject with motor deficits.

Any absolute or relative contra-indication would remain the same across traditional and modern methods. Protocols found effective with the traditional method would apply to the modern, though the modern method would enhance robustness $s$ and reproducibility especially in home or high throughput use.
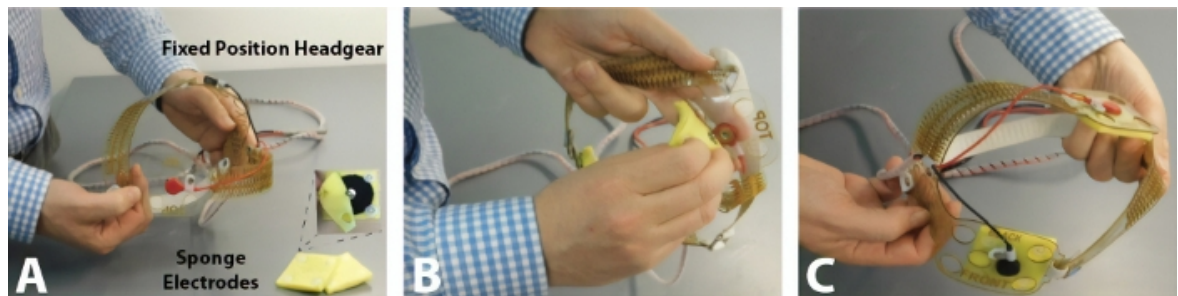

Figure 1: Fixed-position headgear and pre-assembled sponge electrodes. (A) Some fixed-position headgear already include the necessary cables, with pre-assembled sponges designed to snap onto. (B)This figure indicates the headgear setup process by snapping the electrodes firmly in place on to the head strap. (C) Pre-assembled electrodes are already soaked in saline solution. Please click here to view a larger version of this figure. 

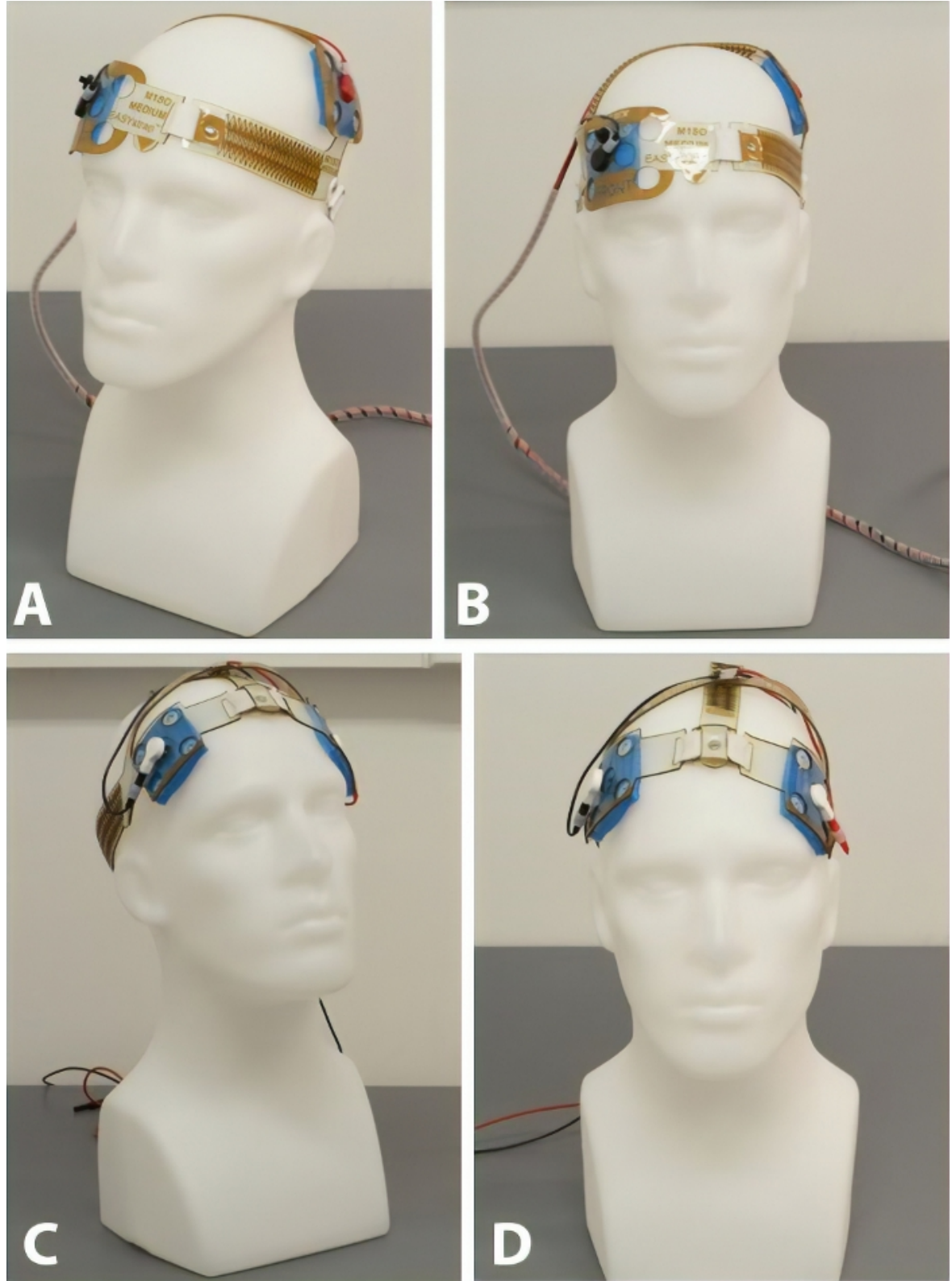

Figure 2: M1-SO montage and Bifrontal montage. (A, B) In the M1-SO montage setup, the anode is placed over the region corresponding to primary motor cortex (M1) and the cathode is placed over the contralateral supra-orbital (SO) region. (A) is the side view and (B) is the front view. (C, D) In the bifrontal montage setup, the anodal electrode is placed over the right and the cathodal electrode is placed over the left dorsolateral prefrontal cortex. (C) is the side view and (D) is the front view. Please click here to view a larger version of this figure. 


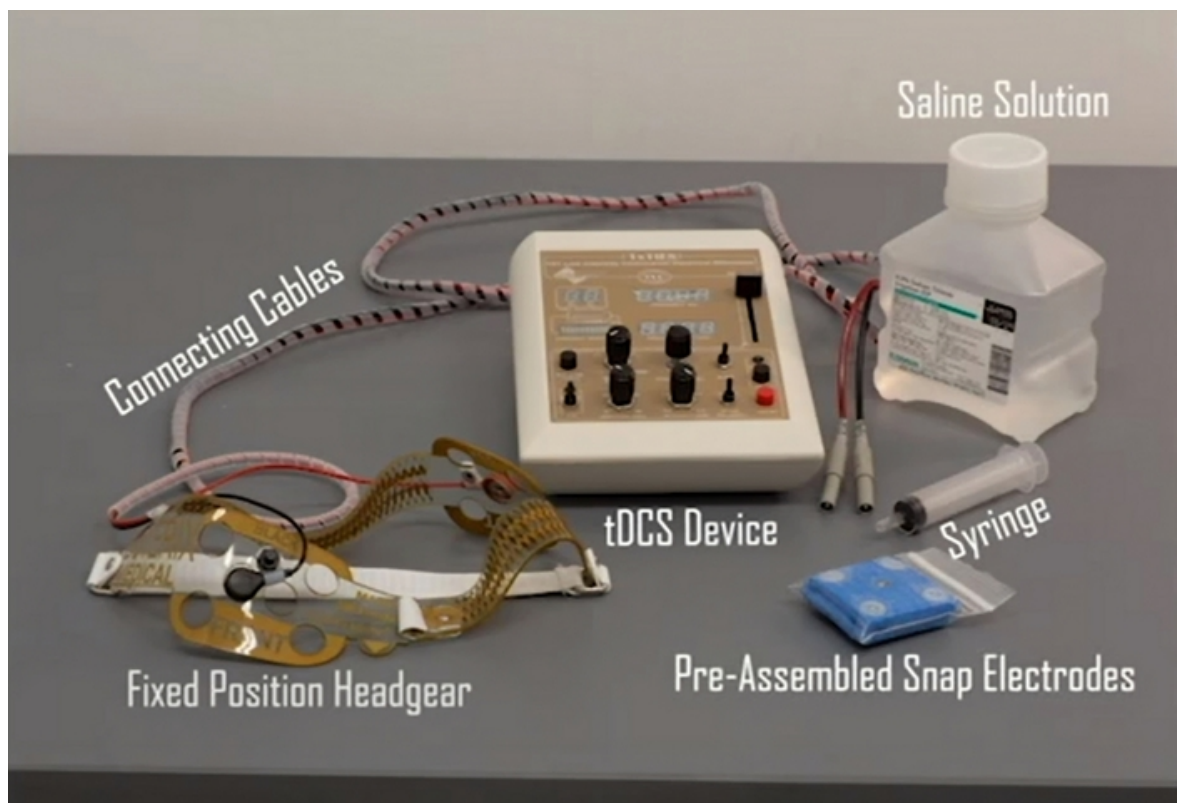

Figure 3: Items that are generally present in every tDCS session. While some materials will depend on the target of the study/treatment, the items listed below are essential for the tDCS session described in this guide. These items include: 1) a tDCS device, 2) single-use snap sponge electrodes, 3) saline solution, 4) a fixed-position headgear (the one below includes the necessary connecting cables), and 5) a syringe for saline application if necessary. Please click here to view a larger version of this figure. 


\section{Traditional tDCS Modern tDCS}
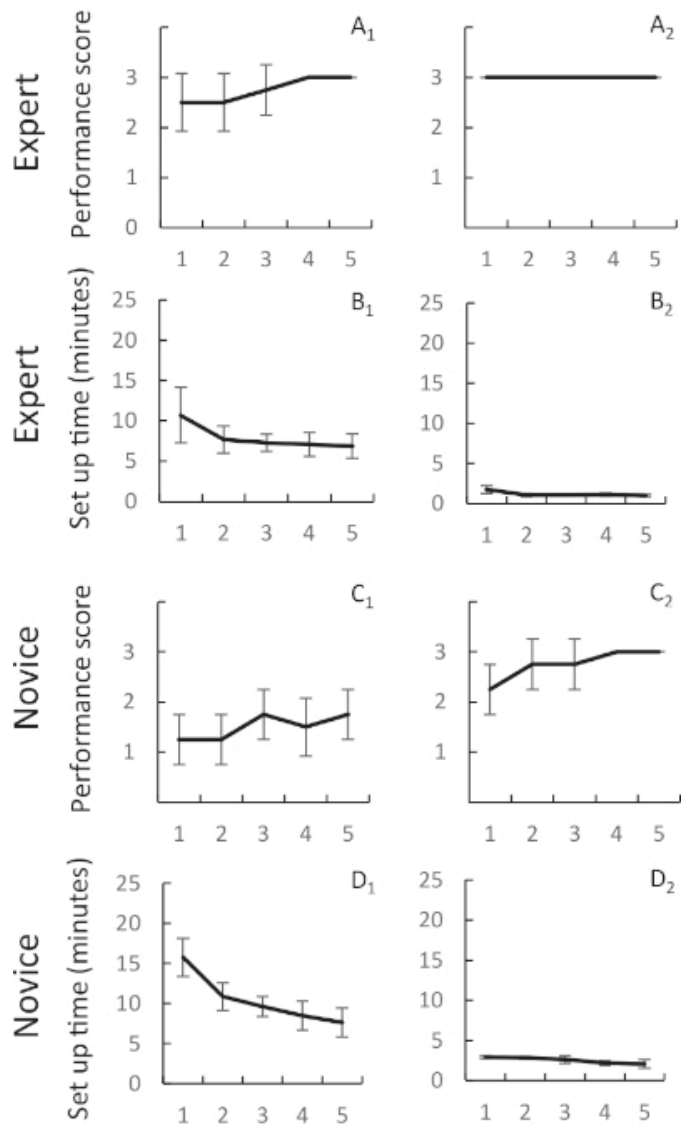

Attempts

Attempts

Figure 4: Setup times and performance scores for novices and experts applying both modern and traditional tDCS method. Expert and novice operators conducted the M1-SO montage setup five times using the traditional tDCS setup method and the modern setup method. The traditional setup method involves taking measurements for the M1-S0 position using the 10-20 EEG system and then placing the electrodes at the target location. For tDCS traditional and modern method, both experts and novices reviewed preparation instructions, as well as additional instructions before the first setup trials. The modern tDCS setup method reduces the setup time and improves performance for both expert and novice subjects because it removes the time-consuming step of the 10-20 EEG measurements for M1-S0 montage. When using the modern tDCS method (Panel $B_{2}$ and $D_{2}$ ), the average setup time taken by the experts and novices was 1.23 minutes $( \pm 0.37)$ and 2.53 minutes $( \pm 0.48)$ respectively. When using the traditional tDCS method (Panel $B_{1}$ and $\left.D_{1}\right)$, the average setup time taken by the experts and novices was 7.93 minutes $( \pm 2.30)$ and 10.47 minutes $( \pm 3.36)$ respectively. After each trial of electrodes setup, performance was measured on a 1-3 scale with 3 scored as error free setup and 1 scored as poor setup. The performance was higher for the modern tDCS method for both experts and novices. For the traditional tDCS method, the average performance by experts and novices was $2.75( \pm 0.25)$ and $1.5( \pm 0.25)$ respectively $\left(P a n e l A_{1}\right.$ and $C_{1}$ ). For the modern tDCS method, the average performance by experts and novices was $3( \pm 0)$ and $2.75( \pm 0.3)$ respectively $\left(\right.$ Panel $A_{2}$ and $\left.C_{2}\right)$. Error bars show standard deviation. Please click here to view a larger version of this figure.

\begin{tabular}{|l|l|l|l|}
\hline & Classical Method & Updated Method & Benefit of Updated Method \\
\hline $\begin{array}{l}\text { Electrode Positioning } \\
\text { Measurement }\end{array}$ & $\begin{array}{l}\text { Multiple tape measures at each } \\
\text { session. }\end{array}$ & $\begin{array}{l}\text { Single tape measure only at first } \\
\text { session. }\end{array}$ & $\begin{array}{l}\text { Decreased time and increased } \\
\text { reliability in electrode positioning. }\end{array}$ \\
\hline Electrode Preparation & $\begin{array}{l}\text { Multiple steps including assembly } \\
\text { and saturation. }\end{array}$ & $\begin{array}{l}\text { No preparation (Pre-saturated). } \\
\text { Includes snap connector. }\end{array}$ & $\begin{array}{l}\text { Decreased time and increased } \\
\text { reliability in electrode preparation. }\end{array}$ \\
\hline Head-gear & $\begin{array}{l}\text { Rubber bands with multiple } \\
\text { connections. }\end{array}$ & $\begin{array}{l}\text { Single head-gear with fixed snap } \\
\text { connector positions. }\end{array}$ & $\begin{array}{l}\text { Decreased time and increased } \\
\text { reliability in electrode positioning. }\end{array}$ \\
\hline
\end{tabular}

Table 1: Summary comparison of classical tDCS method and the modern tDCS method. Regarding electrode position, electrode preparation, and headgear use, the modern tDCS techniques offer advances in reducing time and increasing reliability.

\section{Discussion}

Since 2000, there has been an exponential increase in the rate (number of published trials) and breadth (range of applications and indications) for $t D C S^{5,11,33}$. The modern tDCS protocols illustrated here potentially further supports adoption in human trials, especially of increasing size and sites (e.g., pivotal trials), and ultimately in treatment ${ }^{9}$ as these modern tDCS techniques are simple and normalize critical setup steps. 
Since electrode preparation and position determine tDCS dose ${ }^{12}$, methods to ensure replicable setup underpin reproducible trials. The modern technique described here is expected to be advantageous across inclusion criterion but may provide special benefit in group where conventional techniques prove challenging as a result of scalp/hair conditions, behavior, or in high-throughout (multi-center trials) and remote settings ${ }^{34,35}$. The modern technique, by providing a more secure fixation of the electrodes (e.g. compared to ad hoc elastic straps in the conventional technique) would enhance combination with adjunct behavioral therapies such as mirror therapy ${ }^{36,37,38}$, visual imagery and virtual reality ${ }^{39,40,41}$, or physical therapy ${ }^{34,42,43,44,45}$

tDCS is considered a safe and convenient form of noninvasive brain stimulation ${ }^{5,11}$. Nonetheless, it is still important to ensure that the stimulation is conducted following best practices ${ }^{14}$. All tDCS operators are trained and certified. A detailed study-specific protocol is created outlining any additional materials necessary, the electrode montage used, any tasks if applicable, important safety procedure to be followed before, during, and after the stimulation, as well as study-specific inclusion and exclusion criteria. Some exclusion criteria may include metallic head and/or neck tattoos, metallic implants in head and/or neck, among others - but these are not absolute (e.g. tES in subjects with epilepsy, implant, and acute skull defects) ${ }^{4}$. Many aspects of a tDCS study protocols, such as some materials, electrode placement, duration, among other procedures, are specific to the study design. When modifying the protocol to fit study-specific needs, ensure that those modifications are acceptable to both subject and researcher ${ }^{5,11}$.

A modern tDCS method is described in this guide. This contemporary tDCS application technique is significantly simpler than the conventional method, and so is both faster and less prone to error.

\section{Disclosures}

The City University of New York holds patents on brain stimulation, on which Marom Bikson is an inventor. Marom Bikson is a co-founder of Soterix Medical Inc.

Acknowledgments

This work was supported by the NIH (grants 1R01NS101362-01, 1R01MH111896-01, 1R01NS095123-01, 1R01MH109289-01, 1K01AG050707).

\section{References}

1. Brunoni, A.R. et al. Clinical research with transcranial direct current stimulation (tDCS): challenges and future directions. Brain Stimulation. $\mathbf{5}$ (3), 175-195 (2012).

2. Villamar, M.F., Santos Portilla, A., Fregni, F., Zafonte, R. Noninvasive brain stimulation to modulate neuroplasticity in traumatic brain injury. Neuromodulation: Journal of the International Neuromodulation Society. 15 (4), 326-338 (2012).

3. Datta, A. et al. Gyri-precise head model of transcranial direct current stimulation: improved spatial focality using a ring electrode versus conventional rectangular pad. Brain Stimulation. 2 (4), 201-207, 207.e1 (2009).

4. Huang, Y. et al. Measurements and models of electric fields in the in vivo human brain during transcranial electric stimulation. eLife. 6 (2017).

5. Bikson, M. et al. Safety of Transcranial Direct Current Stimulation: Evidence Based Update 2016. Brain Stimulation. 9 (5), $641-661$ (2016).

6. Nitsche, M.A., Paulus, W. Excitability changes induced in the human motor cortex by weak transcranial direct current stimulation. The Journal of Physiology. 527 Pt 3, 633-639 (2000).

7. Jamil, A. et al. Systematic evaluation of the impact of stimulation intensity on neuroplastic after-effects induced by transcranial direct current stimulation. The Journal of Physiology. 595 (4), 1273-1288 (2017).

8. Monte-Silva, K. et al. Induction of late LTP-like plasticity in the human motor cortex by repeated noninvasive brain stimulation. Brain Stimulation. 6 (3), 424-432 (2013).

9. Lefaucheur, J.P. et al. Evidence-based guidelines on the therapeutic use of transcranial direct current stimulation (tDCS). Clinical Neurophysiology: Official Journal of the International Federation of Clinical Neurophysiology. 128 (1), 56-92 (2017).

10. Buch, E.R. et al. Effects of tDCS on motor learning and memory formation: A consensus and critical position paper. Clinical Neurophysiology: Official Journal of the International Federation of Clinical Neurophysiology. 128 (4), 589-603 (2017).

11. Antal, A. et al. Low intensity transcranial electric stimulation: Safety, ethical, legal regulatory and application guidelines. Clinical Neurophysiology: Official Journal of the International Federation of Clinical Neurophysiology. 128 (9), 1774-1809 (2017).

12. Peterchev, A.V. et al. Fundamentals of transcranial electric and magnetic stimulation dose: definition, selection, and reporting practices. Brain Stimulation. 5 (4), 435-453 (2012).

13. Esmaeilpour, Z. et al. Incomplete evidence that increasing current intensity of tDCS boosts outcomes. Brain Stimulation. 11 (2), $310-321$ (2018).

14. Woods, A.J. et al. A technical guide to tDCS, and related noninvasive brain stimulation tools. Clinical Neurophysiology: Official Journal of the International Federation of Clinical Neurophysiology. 127 (2), 1031-1048 (2016).

15. DaSilva, A.F., Volz, M.S., Bikson, M., Fregni, F. Electrode positioning and montage in transcranial direct current stimulation. Journal of Visualized Experiments. (51) e2744 (2011).

16. Meinzer, M. et al. Transcranial direct current stimulation and simultaneous functional magnetic resonance imaging. Journal of Visualized Experiments. (86), e51730 (2014).

17. Pope, P.A. Modulating Cognition Using Transcranial Direct Current Stimulation of the Cerebellum. Journal of Visualized Experiments. (96) e52302 (2015).

18. Rabau, S. et al. Comparison of the Long-Term Effect of Positioning the Cathode in tDCS in Tinnitus Patients. Frontiers in Aging Neuroscience. 9, 217 (2017).

19. Knotkova, H. et al. Automatic M1-SO Montage Headgear for Transcranial Direct Current Stimulation (TDCS) Suitable for Home and HighThroughput In-Clinic Applications. Neuromodulation: Journal of the International Neuromodulation Society. (2018). 
20. Woods, A.J., Bryant, V., Sacchetti, D., Gervits, F., Hamilton, R. Effects of Electrode Drift in Transcranial Direct Current Stimulation. Brain Stimulation: Basic, Translational, and Clinical Research in Neuromodulation. 10 (1), e1 (2017).

21. Fehér, K.D., Morishima, Y. Concurrent Electroencephalography Recording During Transcranial Alternating Current Stimulation (tACS). Journal of Visualized Experiments. (107), e53527 (2016).

22. Schestatsky, P., Morales-Quezada, L., Fregni, F. Simultaneous EEG Monitoring During Transcranial Direct Current Stimulation. Journal of Visualized Experiments. (76), e50426 (2013).

23. Carvalho, F. et al. Home-Based Transcranial Direct Current Stimulation Device Development: An Updated Protocol Used at Home in Healthy Subjects and Fibromyalgia Patients. Journal of Visualized Experiments. (137), e57614 (2018).

24. Terney, D., Chaieb, L., Moliadze, V., Antal, A., Paulus, W. Increasing human brain excitability by transcranial high-frequency random noise stimulation. The Journal of Neuroscience: The Official Journal of the Society for Neuroscience. 28 (52), 14147-14155 (2008).

25. Guleyupoglu, B., Schestatsky, P., Edwards, D., Fregni, F., Bikson, M. Classification of methods in transcranial electrical stimulation (tES) and evolving strategy from historical approaches to contemporary innovations. Journal of Neuroscience Methods. 219 (2), $297-311$ (2013).

26. Riggs, A. et al. At-Home Transcranial Direct Current Stimulation (tDCS) With Telehealth Support for Symptom Control in Chronically-III Patients With Multiple Symptoms. Frontiers in Behavioral Neuroscience. 12, 93 (2018).

27. Shaw, M.T. et al. Remotely Supervised Transcranial Direct Current Stimulation: An Update on Safety and Tolerability. Journal of Visualized Experiments. (128), e56211 (2017).

28. Brunoni, A.R. et al. The Escitalopram versus Electric Current Therapy for Treating Depression Clinical Study (ELECT-TDCS): rationale and study design of a non-inferiority, triple-arm, placebo-controlled clinical trial. Sao Paulo Medical Journal. 133 (3), 252-263 (2015)

29. Aparício, L.V.M. et al. A Systematic Review on the Acceptability and Tolerability of Transcranial Direct Current Stimulation Treatment in Neuropsychiatry Trials. Brain Stimulation. 9 (5), 671-681 (2016).

30. Ezquerro, F. et al. The Influence of Skin Redness on Blinding in Transcranial Direct Current Stimulation Studies: A Crossover Trial. Neuromodulation: Journal of the International Neuromodulation Society. 20 (3), 248-255 (2017).

31. Brunoni, A. R. et al. A systematic review on reporting and assessment of adverse effects associated with transcranial direct current stimulation. International Journal of Neuropsychopharmacology. 14 (8), 1133-1145 (2011).

32. Shaw, M. et al. Proceedings \#13. Updated Safety and Tolerability of Remotely-Supervised Transcranial Direct Current Stimulation (RS-tDCS). Brain Stimulation: Basic, Translational, and Clinical Research in Neuromodulation. 10 (4), e60-e61 (2017).

33. Grossman, P. et al. transcranial Direct Current Stimulation Studies Open Database (tDCS-OD). bioRxiv. 369215 (2018).

34. Dobbs, B. et al. Generalizing remotely supervised transcranial direct current stimulation (tDCS): feasibility and benefit in Parkinson's disease. Journal of Neuroengineering and Rehabilitation. 15 (1), 11 (2018).

35. Charvet, L. et al. Remotely Supervised Transcranial Direct Current Stimulation Increases the Benefit of At-Home Cognitive Training in Multiple Sclerosis. Neuromodulation: Journal of the International Neuromodulation Society. 21 (4), 383-389 (2018).

36. von Rein, E. et al. Improving motor performance without training: the effect of combining mirror visual feedback with transcranial direct current stimulation. Journal of Neurophysiology. 113 (7), 2383-2389 (2015).

37. Cho, H.S., Cha, H.G. Effect of mirror therapy with tDCS on functional recovery of the upper extremity of stroke patients. Journal of Physical Therapy Science. 27 (4), 1045-1047 (2015).

38. Beaulé, V. et al. Modulation of physiological mirror activity with transcranial direct current stimulation over dorsal premotor cortex. The European Journal of Neuroscience. 44 (9), 2730-2734 (2016).

39. Fuentes, M.A. et al. Combined Transcranial Direct Current Stimulation and Virtual Reality-Based Paradigm for Upper Limb Rehabilitation in Individuals with Restricted Movements. A Feasibility Study with a Chronic Stroke Survivor with Severe Hemiparesis. Journal of Medical Systems. 42 (5), 87-87 (2018).

40. Jax, S.A., Rosa-Leyra, D.L., Coslett, H.B. Enhancing the mirror illusion with transcranial direct current stimulation. Neuropsychologia. 71, 46-51 (2015).

41. Santos, T.E.G. et al. Manipulation of Human Verticality Using High-Definition Transcranial Direct Current Stimulation. Frontiers in Neurology. 9, 825 (2018)

42. Halko, M.A. et al. Neuroplastic changes following rehabilitative training correlate with regional electrical field induced with tDCS. Neurolmage. 57 (3), 885-891 (2011).

43. D'Agata, F. et al. Cognitive and Neurophysiological Effects of Noninvasive Brain Stimulation in Stroke Patients after Motor Rehabilitation Frontiers in Behavioral Neuroscience. 10, 135-135 (2016).

44. Doppelmayr, M., Pixa, N.H., Steinberg, F. Cerebellar, but not Motor or Parietal, High-Density Anodal Transcranial Direct Current Stimulation Facilitates Motor Adaptation. Journal of the International Neuropsychological Society: JINS. 22 (9), 928-936 (2016).

45. Bowling, N.C., Banissy, M.J. Modulating vicarious tactile perception with transcranial electrical current stimulation. The European Journal of Neuroscience. 46 (8), 2355-2364 (2017). 